\title{
BMJ Open Protocol for the cost-effectiveness of parenteral iron and oral iron therapy for first-line management of anaemia among pregnant women in a natural programme setting in Gujarat
}

\author{
Somen Saha (D) ,' Bharat Desai (D) , ${ }^{1}$ Manish Fancy, ${ }^{2}$ Tapasvi Puwar, ${ }^{1}$ \\ Deepak Saxena, ${ }^{1}$ Komal Shah, ${ }^{1}$ Apurvakumar Pandya (iD ${ }^{1}$
}

To cite: Saha S, Desai B, Fancy M, et al. Protocol for the cost-effectiveness of parenteral iron and oral iron therapy for first-line management of anaemia among pregnant women in a natural programme setting in Gujarat. BMJ Open 2021;11:e044712. doi:10.1136/ bmjopen-2020-044712

- Prepublication history for this paper is available online. To view these files, please visit the journal online (http://dx.doi. org/10.1136/bmjopen-2020044712).

Received 18 September 2020 Accepted 02 June 2021

\section{ABSTRACT}

Introduction Maternal anaemia is a major public health issue in India. The government of India recommends parenteral iron to manage moderate and severe grades of anaemia. In contrast to its clinical efficacy, the costeffectiveness of intravenous iron sucrose and ferric carboxymaltose is not yet established in Indian context. This article illustrates the protocol of health technology assessment to evaluate the cost-effectiveness of intravenous therapy on the improvement of haemoglobin concentration over oral therapy.

Methods and analysis The study will be carried out in two districts of Gujarat state. The study participants will be selected by a proportionate sampling method from the rural, tribal, desert and coastal region of the districts. Data will be collected over 1 year on key outcome indicators using a mixed-method approach. Key informant interviews will be conducted, and cost data will be gathered to perform cost-effectiveness analysis.

Ethics and dissemination This study is approved by the Technical Appraisal Committee of Health Technology Assessment India, Department of Health Research and Institutional Ethics Committee of the Indian Institute of Public Health, Gandhinagar.

\section{INTRODUCTION}

Maternal anaemia is a major public health issue in India. Specifically, iron-deficiency anaemia (IDA) during pregnancy is a significant public health concern as it is associated with perinatal mortality, preterm birth, neonatal low-birth-weight, and maternal mortality and morbidity. ${ }^{1}$ Furthermore, in Gujarat, iron deficiency is higher $(65 \%)$ among anaemic women. ${ }^{2}$ Conventionally, the first line of treatment for IDA in pregnant women is oral iron (OI) therapy. Nevertheless, OI therapy is associated with numerous side effects, chiefly constipation, vomiting and epigastric discomfort. Therefore, compliance with this mode of treatment is a major
Strengths and limitations of this study

- The study will be conducted in a natural programme setting at the Peripheral Health Institutes.

- Iron therapy will be given to all patients irrespective of their iron store status as iron-deficiency prevalence is higher among anaemic women in Gujarat.

- The cost-effectiveness analysis plan, including incremental cost-effectiveness ratio calculations for the two interventions, will be compared, and subgroup analyses will be carried out.

- A digital haemoglobinometer will be used for haemoglobin estimation.

There might be a problem of attrition.

concern. ${ }^{3}$ Evidence reveals that OI is not sufficient for the treatment of moderate and severe IDA detected during the late stage of pregnancy. ${ }^{4}$

Of all the parenteral iron preparations available, intravenous administration has emerged as an effective alternative to OI therapy in pregnant women. ${ }^{5}$ Apart from its quick absorption, intravenous mode is also known to impart a lesser incidence of hypersensitive reactions. ${ }^{6}$ Additionally, intravenous iron sucrose (IVIS) has been reported to be safe and effective during pregnancy. ${ }^{7-9}$ Recently, ferric carboxy maltose (FCM) is also considered as an effective treatment for IDA during pregnancy and the postpartum periods. ${ }^{1011}$ Table 1 shows national guidelines for IDA treatment among pregnant women.

Prime Minister's Overarching Scheme for Holistic Nourishment Campaign (POSHAN Abhiyaan) was launched in March 2018 in India to reduce the burden of anaemia. ${ }^{12}$ Complying with the overarching objective of POSHAN Abhiyaan, the Anaemia Mukt Bharat (Anaemia-Free India) strategy was
Correspondence to

Somen Saha; ssaha@iiphg.org 
Table 1 I-NIPI operational guidelines for management of iron deficiency anaemia among pregnant women

For mild (10-10.9 g/dL) Two tablets of iron and folic acid and moderate $\left(7-9.9 \mathrm{~g} /\right.$ tablet, that is, OI $\left(60 \mathrm{mg}^{*}\right.$ elemental $\mathrm{dL}$ ) anaemia iron and $500 \mu \mathrm{g}$ folic acid) daily, orally given by the health provider during the antenatal care (ANC) contact.

Parenteral iron therapy (IVIS or FCM) may be considered the first line of management in pregnant women who are detected to be anaemic late in pregnancy or compliance is likely to be low (high chance of loss to follow-up).

$\begin{array}{ll}\text { For severe }(5-6.9 \mathrm{~g} / \mathrm{dL}) & \text { The treatment will be done using } \\ \text { anaemia } & \text { IVIS or FCM by the medical officer. } \\ & \text { Immediate hospitalisation is } \\ & \text { recommended in the third trimester } \\ & \text { of pregnancy at a health facility } \\ & \text { where round-the-clock specialist } \\ & \text { care is available. }\end{array}$

Source: I-NIPI guideline (MoHFW, GOI 2018)..$^{13}$ *100 mg elemental iron is being supplied by the government, and hence it is being used instead of $60 \mathrm{mg}$. FCM, ferric carboxymaltose; I-NIPI, Intensified National Iron Plus Initiative; IVIS, intravenous iron sucrose; OI, oral iron.

designed to achieve the ambitious target of $50 \%$ reduction of anaemia among women of reproductive age by $2025 .{ }^{13}$ To fulfil this target, digital methods for testing and point-of-care treatment have been recommended.

Although IVIS has a limited maximum permissible dose per week, requiring multiple visits to deliver the required iron dose, FCM can be administered in a more considerable amount. Some randomised controlled trials ${ }^{14-16}$ have shown promising results with IVIS and FCM. However, no studies compare the cost-effectiveness of IVIS and FCM with the OI therapy on improvement of haemoglobin $(\mathrm{Hb})$ level in programmatic settings. Hence, this study compares the cost-effectiveness of the IVIS and FCM therapy against OI among pregnant women with IDA in a programmatic setting of Banaskantha and Devbhoomi Dwarka districts of Gujarat, India.

\section{METHODS}

\section{Study population}

The observational study will be undertaken prospectively at Banaskantha and Devbhoomi Dwarka districts of Gujarat during 2020-2021. All registered pregnant women between 14 and 18 weeks of gestation period will be enrolled from both districts for 7-8 months in the study. During the study period, patients with moderate $(\mathrm{Hb}<10 \mathrm{~g} / \mathrm{dL})$ and severe $(\mathrm{Hb}<7 \mathrm{~g} / \mathrm{dL})$ anaemia will be recruited. The study will follow a natural programme setting without manipulating the study environment. Classification and treatment of IDA among pregnant women will be as per national guidelines (table 1). Complying the government guidelines, ${ }^{13}$ the medical officer at the healthcare facility will determine the appropriate iron therapy assignment based on the participant's anaemia status in their routine practice. Patients with moderate anaemia will receive OI, while patients with severe anaemia and moderate anaemia with very low compliance or a high chance of loss-tofollow-up will be provided parenteral iron therapy, either IVIS or FCM. Patients who received a blood transfusion in the last 120 days or required a blood transfusion at any stage of the intervention will be excluded from the study. Apart from these, patients with haemoglobinopathy, other red cell disorders or any chronic infections such as hepatitis, HIV, and showing any history of an allergic reaction to intravenous iron infusion will also be excluded from the study. Key statistics of the study districts, namely, Banaskantha and Devbhoomi Dwarka, are presented in table 2 .

\section{Sample size}

From an earlier study, ${ }^{15}$ the $0.75 \mathrm{~g} / \mathrm{dL}$ change in $\mathrm{Hb}$ between these two groups is considered to calculate the sample size. Thus, by considering $\alpha$ error $5 \%$ and the power of the study as $95 \%$, the estimated sample size was 26 per group. By assuming a loss-to-follow-up of $20 \%$, the expected sample size comes to 32. Thus, a total of 64 patients (32 in each group) will be selected from the Devbhoomi Dwarka district. Considering the demography of Banaskantha and patients' higher enrolment, we decided to take 100 patients in each group. Hence, a total of 200 patients will be selected for the Banaskantha district study. Injection (inj.) FCM has not yet started in Banaskantha and Devbhoomi Dwarka districts. Therefore, the inclusion of a third group (inj. FCM) will depend on the availability and coverage of inj. FCM in the respective districts. We expect to enrol 396 patients in the study from October 2020 to achieve the expected sample size.

Table 2 Key statistics of Banaskantha and Devbhoomi Dwarka districts based on 2019-2020 information

\begin{tabular}{|c|c|c|}
\hline Estimated numbers & Banaskantha & $\begin{array}{l}\text { Devbhoomi } \\
\text { Dwarka }\end{array}$ \\
\hline Total pregnancy registered & 40067 & 7015 \\
\hline $\begin{array}{l}\text { Total anaemic pregnant } \\
\text { women }\end{array}$ & 19858 & 4213 \\
\hline $\begin{array}{l}\text { Pregnant women with } \\
\text { moderate anaemia (study } \\
\text { cohort) }\end{array}$ & 13276 & 1363 \\
\hline $\begin{array}{l}\text { Pregnant women with } \\
\text { severe anaemia (study } \\
\text { cohort) }\end{array}$ & 6582 & 32 \\
\hline
\end{tabular}

Source: Records maintained by District Health Team for the year (April 2019-March 2020). 


\section{Study procedures}

The study will be conducted in a natural programme setting of the primary health centres (PHCs). The blocks will be selected by proportionate sampling method from the four regions of the district, that is, rural, tribal, desert and coastal regions, based on the previous year's registered pregnancy. The PHCs will be selected randomly from the proportionately selected blocks. Patients with moderate anaemia who are exclusively on OI supplements will be recruited as the control population. Women will be asked to bring back empty packs and be asked about the intake of tablets and the stools' colour to ensure consumption of the tablets. Patients indicated for IVIS or FCM by the medical officer will be taken under the intervention arm. Iron requirements will be calculated using modified Ganzoni's formula as follow. ${ }^{16}$

Iron requirement $(\mathrm{mg})$ : Total iron deficit $[\mathrm{mg}]=$ Bodyweight $[\mathrm{kg}]$ $\times($ target $\mathrm{Hb}-$ actual $\mathrm{Hb})\left[\frac{\mathrm{g}}{\mathrm{dl}}\right] \times 0.24+$ storage iron $(500)[\mathrm{mg}]$

At the baseline, information regarding sociodemographic profile, obstetric history, preintervention assessment (includes height, weight and $\mathrm{Hb}$ ) and history of intervention will be recorded. All pregnant women will be followed up to 6 weeks following delivery. Hb levels will be measured at the end of each month after baseline and at the 42nd day of the postnatal period. $\mathrm{Hb}$ estimation will be carried out by a digital haemoglobinometer (HemoCue $\mathrm{Hb} \mathrm{201}{ }^{+}$System recommended by the Ministry of Health and Family Welfare $)^{13}$ through a laboratory technician available at the facility before treatment and during each follow-up visit. Figure 1 presents a summary of the study plan.

\section{Valuing of health outcomes}

Health-related quality of life will be assessed using the EuroQol-5 Dimensions-5 Levels (EQ-5D-5L) tool at baseline and during each follow-up visit. Five domains, namely, mobility, self-care, usual activities, pain and discomfort, and anxiety and depression, will be assessed on five-level scores ranging from 1 to 5 , with 1 being the worst and 5 the best. The response on the EQ-5D-5L will be converted into a single index value to derive quality-adjusted lifeyear (QALY). The key clinical outcome will be assessed by a mean increase in $\mathrm{Hb}$ level.

\section{Measuring the cost of care}

The per beneficiary cost of therapy will be estimated from a societal perspective. Cost under various heads such as cost of therapy, consumables, healthcare resources (shared human resource, beds, etc), out-ofpocket expenses and wage loss will be collected from financial records and field interviews. Cost of therapy by OI, IVIS and FCM will be obtained from both government rate contracts and local bulk procurement as the case be. Details of consumables, including materials and supplies issued, consumed, quantity used per test and price per unit, will be collected from the respective facility. Financial and administrative records, including procurement and consumables records, will be reviewed. Healthcare facility-related costs such as time and bed occupied during IVIS or FCM administration will be calculated, whereas research costs such as costs associated with additional testing of sampled cases using digital haemoglobinometer will be excluded from the analysis. Out-of-pocket expenditure in terms of travelling cost and wage loss (of a patient and accompanying person) due to referral or follow-up will be gathered from field records. The costs will be presented as average values across interventions, viz-OI, IVIS or FCM.

\section{Data storage and security}

Data will be stored in encrypted and password-protected computer system at the institution. Hard copy of the records will be stored in a locked cupboard in a secure location at the institute. Access to records and study data will be restricted to study personnel. Study data will be de-identified and will be stored separately from the data.

\section{Patient and public involvement}

There was no patient or public involvement in the design and planning of the study.

\section{PLAN OF ANALYSES}

The primary analysis will be reported as per intention to treat' to assess the clinical effectiveness and avoid potential bias due to the exclusion of patients. In contrast, the secondary analysis will be carried out as per 'per protocol' analysis to identify a treatment effect under optimal conditions. The primary outcome measure will be the mean change in $\mathrm{Hb}$ level from baseline. Besides, secondary outcome measures will include target $\mathrm{Hb}$ achievement $(>11 \mathrm{~g} / \mathrm{dL})$, the incidence of treatment failure including change of therapy for side effects, maternal mortality due to postpartum haemorrhage $(\mathrm{PPH})$, pregnancy-related complications due to PPH, preterm birth, low-birth-weight neonates, maternal weight gain, early neonatal mortality and requirement of blood units during delivery. The analysis will be adjusted for protein-energy malnutrition, gestation, compliance to treatment, socioeconomical background, parity and blood loss during delivery. Findings will be subgrouped according to population (urban/ $\mathrm{rural} / \mathrm{tribal} /$ coastal), baseline $\mathrm{Hb}$ levels, trimester of pregnancy, grades of compliance, duration and treatment dose. Further subgroup analysis will be employed within the intervention arm to understand the doseresponse and mean change in $\mathrm{Hb}$.

\section{Cost-effectiveness analysis}

The cost-effectiveness analysis (CEA) per QALY gained will be undertaken by estimating the programme 


\begin{tabular}{|l|}
\hline Cost from societal perspective \\
- Cost of administering IVIS \\
- Cost of administering FCM \\
- Time spend by health workers \\
including bed occupancy \\
- Transportation cost
\end{tabular}

Figure 1 Summary of the study design to compare the cost-effectiveness of the IVIS and FCM therapy among pregnant women with IDA. FCM, ferric carboxymaltose; Hb, haemoglobin; IDA, iron-deficiency anaemia; inj. injection; IVIS, intravenous iron sucrose.

implementation cost from the health system and societal perspective. The incremental cost-effectiveness ratio (ICER) will be calculated by combining costs and outcomes. The CEA results will be expressed in cost per patient achieving target $\mathrm{Hb}$ across the approaches (OI, IVIS and FCM) and cost per unit change in mean $\mathrm{Hb}$ level after 6 weeks of delivery compliance rate.

The cost-effectiveness model will be cross-verified using subgroup analysis, where the population will be grouped according to landscape classification (urban/ rural/tribal/coastal), baseline $\mathrm{Hb}$ level, trimester of pregnancy and compliance. One-way sensitivity analysis will be carried out by varying model parameters to estimate joint uncertainty in all parameters. A tornado chart will be presented using ICER values to depict changes in selected variables that influence the results.

\section{DISCUSSION}

It is evidenced that with an increase in each $1 \mathrm{~g} / \mathrm{dL}$ mean $\mathrm{Hb}$ level, the risk of maternal mortality falls by $25 \%{ }^{17}$ Evidence indicates that the $\mathrm{Hb}$ level in the range between $5 \mathrm{~g} / \mathrm{dL}$ and $12 \mathrm{~g} / \mathrm{dL}$ is significantly associated with reducing maternal mortality. ${ }^{18}$ Therefore, 
an aggressive approach for treating moderate and severe anaemia among pregnant women is justified. OI therapy is indicated in a patient diagnosed with moderate anaemia in pregnancy unless the patient presents late in pregnancy or those whose compliance is likely to be low (high chance of loss-to-follow up). In these patients, parenteral iron therapy is indicated. A systemic review conducted by Qassim et al in $2018^{8}$ concluded that different intravenous iron preparations (FCM/iron polymaltose/iron sucrose) were similar in terms of safety and efficacy. They documented that cost and convenience of administration mainly influence the selection of specific parenteral iron preparation. However, evidence on the effect of parenteral iron therapy on improvements in critical maternal or perinatal outcomes lacked in the available literature.

Unfortunately, no studies from Indian settings had performed comprehensive cost (including cost incurred by health system) evaluation of oral and parenteral iron therapy. ${ }^{19}$ One cost-effectiveness study conducted in Uttar Pradesh between oral versus injectable iron therapy was primarily hospital-based, and health system cost was not included. ${ }^{19}$ Similarly, other studies conducted by Jose $e t a l^{14}$ and Mahey $e t a l^{20}$ had also excluded health system cost in CEA. In conclusion, this study will be conducted at Peripheral Health Institutes in a natural programme setting using a health system and societal perspective. Therefore, it has a huge scope of generating evidence for policy-makers.

\section{ETHICS AND DISSEMINATION}

Technical approval has been obtained from the Technical Appraisal Committee of the Department of Health Research and the Indian Institute of Public Health Gandhinagar's institutional ethics committee (TRCIEC No: 11/2019-20). A manuscript with the results of the primary study will be published in a peer-reviewed journal.

\section{Twitter Apurvakumar Pandya @apurva_b_pandya}

Acknowledgements This study is proposed to be conducted in collaboration with the Health and Family Welfare Department, Government of Gujarat and Department of Health Research, Ministry of Health and Family Welfare, Government of India.

Contributors SS: conceptualised and designed the study; BD: drafted the protocol; AP: aided manuscript writing, review and editing. DS,TP, MF and KS: reviewed the content of the protocol and edited the manuscript. All authors read and approved the manuscript.

Funding This study will be conducted by the Regional Resource Centre for Health Technology Assessment established at Indian Institute of Public Health, Gandhinagar (IIPHG) with support from the Department of Health and Research, Ministry of Health and Family Welfare, Government of India (grant number T.11011/12/2019-HR/E-Office- 3207453 dated 23 June 2020).

\section{Competing interests None declared.}

Patient and public involvement Patients and/or the public were not involved in the design, or conduct, or reporting, or dissemination plans of this research.

Patient consent for publication Not required.

Provenance and peer review Not commissioned; externally peer reviewed.
Open access This is an open access article distributed in accordance with the Creative Commons Attribution Non Commercial (CC BY-NC 4.0) license, which permits others to distribute, remix, adapt, build upon this work non-commercially, and license their derivative works on different terms, provided the original work is properly cited, appropriate credit is given, any changes made indicated, and the use is non-commercial. See: http://creativecommons.org/licenses/by-nc/4.0/.

\section{ORCID iDs}

Somen Saha http://orcid.org/0000-0002-8043-3388

Bharat Desai http://orcid.org/0000-0003-1978-547X

Apurvakumar Pandya http://orcid.org/0000-0003-0178-3978

\section{REFERENCES}

1 Kumari S, Garg N, Kumar A, et al. Maternal and severe anaemia in delivering women is associated with risk of preterm and low birth weight: a cross sectional study from Jharkhand, India. One Health 2019;8:100098.

2 Jasani J, Trivedi J, Rajdev S. Hematological study of iron deficiency anaemia in pregnancy in central Gujarat. Int J Biomed Adv Res 2019;10:7-9.

3 Tolkien Z, Stecher L, Mander AP, et al. Ferrous sulfate supplementation causes significant gastrointestinal side-effects in adults: a systematic review and meta-analysis. PLoS One 2015;10:e0117383.

4 Marahatta R. Study of anaemia in pregnancy and its outcome in Nepal medical college teaching Hospital, Kathmandu, Nepal. Nepal Med Coll J 2007;9:270-4

5 Kalaivani K. Prevalence \& consequences of anaemia in pregnancy. Indian J Med Res 2009;130:627-33.

6 Kriplani A, Mahey R, Dash BB, et al. Intravenous iron sucrose therapy for moderate to severe anaemia in pregnancy. Indian $\mathrm{J}$ Med Res 2013;138:78-82.

7 Neogi SB, Devasenapathy N, Singh R, et al. Safety and effectiveness of intravenous iron sucrose versus standard oral iron therapy in pregnant women with moderate-to-severe anaemia in India: a multicentre, open-label, phase 3, randomised, controlled trial. Lancet Glob Health 2019;7:e1706-16.

8 Qassim A, Mol BW, Grivell RM, et al. Safety and efficacy of intravenous iron polymaltose, iron sucrose and ferric carboxymaltose in pregnancy: a systematic review. Aust N Z J Obstet Gynaecol 2018;58:22-39.

9 Dubey S, Suri V, Aggarawal N, et al. Is it safe to use intravenous iron sucrose during pregnancy? A randomized controlled trial. Int J Reprod Contracept Obstet Gynecol 2013;2:544.

10 Rathod S, Samal SK, Mahapatra PC, Samal S, et al. Ferric carboxymaltose: a revolution in the treatment of postpartum anemia in Indian women. Int J Appl Basic Med Res 2015;5:25-30.

11 Khalafallah AA, Dennis AE. Iron deficiency anaemia in pregnancy and postpartum: pathophysiology and effect of oral versus intravenous iron therapy. J Pregnancy 2012;2012:1-10.

12 Ministry of Women and Child Development Department, Government of India. Jan Andolan guidelines- Poshan Abhiyan, 2019.

13 Ministry of Health and Family Welfare, Government of India. Intensified national iron plus initiative (I-NIPI): operational guidelines for programme managers, 2018.

14 Jose A, Mahey R, Sharma JB, et al. Comparison of ferric carboxymaltose and iron sucrose complex for treatment of iron deficiency anemia in pregnancy- randomised controlled trial. BMC Pregnancy Childbirth 2019;19:54.

15 Neeru S, Nair NS, Rai L. Iron sucrose versus oral iron therapy in pregnancy anemia. Indian J Community Med 2012;37:214-8.

16 Ganzoni AM. [Intravenous iron-dextran: therapeutic and experimental possibilities]. Schweiz Med Wochenschr 1970;100:301-3.

17 Ezzati M, Lopez AD, Rodgers AA. Comparative quantification of health risks: global and regional burden of disease attributable to selected major risk factors, 2004.

18 Kant S. Do we have a magic bullet to treat moderate and severe anemia in pregnant women? Indian J Public Health 2019;63:165-70.

19 Maheshwari B, Mahtab V, Tyagi S. Evaluation of efficacy, safety and cost-effectiveness of oral iron and injectable iron sucrose and ferric carboxy maltose in pregnant women in 2 nd and 3 rd trimester in anaemia. Indian J Obs Gynecol Res 2017;4:96-100.

20 Mahey R, Kriplani A, Mogili KD, et al. Randomized controlled trial comparing ferric carboxymaltose and iron sucrose for treatment of iron deficiency anemia due to abnormal uterine bleeding. Int $\mathrm{J}$ Gynaecol Obstet 2016;133:43-8 https://obgyn.onlinelibrary.wiley. com/doi/abs/10.1016/j.ijgo.2015.09.007 